\title{
面向拔尖创新人才培养，革新分析化学课程教学
}

郭慧敏 ${ }^{*}$, 吴硕 ${ }^{*}$

大连理工大学化工学院化学系, 辽宁 大连 10023

摘要: 详细介绍了为适应拔尖创新人才培养的要求, 我校应用化学(理科)专业分析化学课程教学中通过优化教学内 容, 实施 “以学生为中心、问题为导向” 的教学设计, 让学生体验 “在学中用、在用中学” 的主动学习而采取的具 体举措和思考。

关键词: 教学设计; 主动学习; 分析化学; 拔尖创新人才

中图分类号: G64; O6

\section{Reform Analytical Chemistry Course for Cultivation of Top Innovative Talents}

\author{
Huimin Guo *, Shuo Wu * \\ Department of Chemistry, School of Chemical Engineering, Dalian University of Technology, Dalian 116024, \\ Liaoning Province, China.
}

\begin{abstract}
For the cultivation of top innovative talents at Dalian University of Technology in recent years, this paper elaborates the reflections and detailed procedures taken in reform of analytical chemistry course by reorganization of course contents, realization of student-oriented and question-oriented instructional design for the students to experience active leaning through Do-in-Learning and Learn-by-Doing.
\end{abstract}

Key Words: Instructional design; Active leaning; Analytical chemistry; Top innovative talents

培养拔尖创新人才是高等教育的战略目标之一。教育部早在 2009 年就启动了 “基础学科拔尖学 生培养试验计划”, 各兄弟院校均已开展了相关实践, 尤其在化学学科中成果已经不断展现 ${ }^{[1-9] 。 2020}$ 年1月, 作为国家选拔和培养基础学科拔尖人才以及改革高校录取选拔方式的最新举措, 教育部为了 选拔培养有志于服务国家重大战略需求, 且综合素质优秀的学生, 突出基础学科在未来科技发展和 创新中的支撑引领作用 ${ }^{[10]}$, 又公布了《在部分高校开展基础学科招生改革试点工作的建议》(也称 “强 基计划” ), 宣布以36所 “双一流” 建设高校为试点, 招收化学等基础学科方面的拔尖创新人才。

大连理工大学应用化学(理学)专业始创于1987年, 一直围绕 “面向未来、适应需求、引领发展、 理念先进、保障有力” 的一流专业建设要求开展办学, 是我校理科人才培养的高地 ${ }^{[11]}$ 。2010年改称 化学基础科学班, 2013年开始与中国科学院大连化学物理研究所协同办学, 改称张大显化学菁英班。 我校应用化学(理学)专业于 2015 年被评为首批辽宁省普通高等学校本科优势特色专业并名列榜 首, 2018年被评为辽宁省普通高等院校首批一流本科教育示范专业, 2020 年成为教育部 “双万计

收稿: 2021-07-05; 录用: 2021-08-11; 网络发表: 2021-08-24

*通讯作者, Emails: guohm@dlut.edu.cn (郭慧敏); wushuo@dlut.edu.cn (吴硕)

基金资助：国家本科教学工程项目 “分析化学一流学科课程建设和大连理工大学研究生教学改革基金项目” (jg2017016) 
划” 首批国家级一流本科专业建设点和教育部 “强基计划” 首批招生改革试点专业, 并已于 2020 年 开始 “强基计划” 招生 ${ }^{[12]}$ 。从近几年学生情况来看, 我校应用化学(理学)专业的学生综合素质过硬, 高中阶段的相关学科基础比较扎实。教学环节中面临的主要问题是如何将他们培养成拔尖创新人才。 解决这个问题不仅需要将拔尖创新人才培养的理念落实到课程教学中, 也需要对教学团队以及课程 教学进行优化调整。这种调整不仅需要优化教学内容、强化基础理论教学, 更要从教学内容的广度 和深度上进行挖掘和拓展, 在 “以学生为中心” 、理论与实践相结合的教学中深化学生对理论的理 解, 培养他们的思辨能力, 以实现拔尖创新人才培养的目标 ${ }^{[12]}$ 。

分析化学是理科化学专业四大基础课之一, 内容包括化学分析和仪器分析两部分。我国高校理 科化学专业通常以分析化学作为基础, 分别开设分析化学和仪器分析两门课, 二者互为补充。分析 化学主要教学内容包括四大滴定分析法、重量分析法、简单的仪器分析(如: 分光光度法)和误差分析 与数据处理等, 具有概念与原理的理论性强等突出的特点 ${ }^{[13,14]}$ 。在以往教学中, 我们发现传统教学 模式往往使学生处于被动学习的状态, 学生会感觉内容较为枯燥、难以理解透彻, 进而逐渐失去理 论课学习的热情。尽管现在有各种辅助在线教学资源和新颖的教学工具, 但效果仍有很大提升空 间 ${ }^{[15]}$ 。近年来, 我们 “以学生为中心”, 根据拔尖创新人才培养的目标, 通过不断探索, 逐步调整 优化了分析化学理论课教学内容、革新教学模式, 教学效果得到显著提高。本文总结了近几年我校 应用化学(理科)专业分析化学课程教学中采取的一些措施。

\section{1 问题导向的教学设计, “在用中学, 在学中用”}

我校应用化学专业本科生在中学阶段均是综合素质优秀的拔尖学生, 他们成长于我国经济持续 快速增长、科技发展日新月异的新世纪, 视野开阔、接受能力强, 他们的学习渠道己不局限于读书 和课堂。在中学学习过程中, 他们往往只是被动地接受和认知来自各方和各种形式信息, 因此在一 定程度上缺少对化学理论深入认知基础上的主动思考和批判性思维。为了适应新时代对拔尖人才的 培养需求, 我们尝试从根本上将课堂角色进行转变入手, 进行 “以学生为中心、问题为导向” 的教 学设计, 在强化基础理论教学的同时引导学生去主动思考, “在用中学, 在学中用”, 夯实理论基础 的同时, 也培养学生利用所学理论进行批判性思维的能力。

分析化学主要教学内容包括四大滴定分析法、重量分析法、简单的仪器分析(如: 分光光度法)和

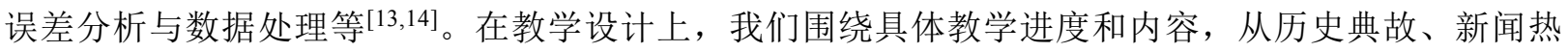
点或生活常识中, 选择具有正能量、科学性和时代特征的内容, 进行 “问题” 设计。通过对现有的 分析化学教学内容进行深入挖掘和整理后, 我们发现几乎每节课都有合适的结合实际的切入点。 表1中给出了教学中设计的部分 “问题”。最理想的 “问题” 应该涵盖知识点涉及的核心教学内容, 在讲述 “问题” 过程中引出教学内容, 把知识点融入到解决 “问题” 过程中, 实现 “在用中学” 。 这样做的好处在于将学生的注意力带入到课堂教学内容中, 利用 “问题” 引导学生主动思考, 通过 “打怪过关” 的学习体验来提高学生的学习热情。在教学过程中, 学生也容易把教学内容和先修理论 结合起来, 提升对知识点的理解。这样的教学过程还具有规范性和逻辑性特点, 起到强化逻辑思维、 传授学习方法的作用, 对学生在今后科研工作中观察问题、分析和解决问题都有很大好处。为了方 便学生复习、反思, 会在课堂结束前, 对教学内容重点进行总结, 并在条件允许的时候考查学生的 理解情况。

对于理论教学内容, 我们先由简入繁地引导学生利用先修课程的理论, 围绕 “问题” 和教学内 容进行思考, 并且抓重点和难点进行细节讲解, 保持循序渐进、“在用中学” 的学习体验, 最后在 实践案例分析中再进行提升, 实现 “在学中用”。化学本来就是从实验研究发展起来的科学。在具体 教学内容设计上, 我们将分析化学理论与配套实验教学内容紧扣, 通过实验案例将理论具体化, 实 现理论与实践相结合, 让学生在课内就把化学理论应用起来。此外, 分析化学还是一门已经渗透到 我们衣食住行各个方面的一门科学, 除实验教学相关的内容外, 我们还把生活中的分析化学理论的 
应用案例也融入到教学内容中。例如, 在络合滴定法教学中, 我们会介绍水的总硬度测定的EDTA法。 教学中会要求学生首先思考水样总硬度的成因和表示方法, 在介绍指示剂铬黑 $\mathrm{T}$ 和钙指示剂等金属 指示剂的特点和作用之后再介绍EDTA与钲、镁离子的络合平衡, 引导学生思考钙、镁离子与指示剂 和EDTA之间络合平衡和竞争关系, 从而引出EDTA法测定水中总硬度的原理和方法。最后, 再引导 学生去分析如温度、测试时间等因素对测量结果的影响。因为课时有限, 一些知识点没办法举一反 三。为此, 我们将课堂教学内容和生活中的小问题、小常识联系起来, 提出例如食醋中的酸浓度等 问题, 让学生在课下去思考可能的解决途径, 让学生了解分析化学无处不在, 需要利用科学的方法 探索真相。此外, 我们还选取了一些被广泛关注的包含分析化学内涵的社会问题, 例如饮用水质量 和环境检测等, 引导学生去利用所学理论进行思考, 提高他们对学科理论的认同感并感受化学理论 旺盛的生命力。

表1 针对部分章节教学内容的 “问题” 设计举例

\begin{tabular}{|c|c|c|}
\hline 部分章节 & 内容 & 部分“问题”举例 \\
\hline 酸碱滴定法 & 酸碱溶液的pH计算 & $\mathrm{pH}$ 试纸和 $\mathrm{pH}$ 计测量结果与根据溶液浓度计算得到的 $\mathrm{pH}$ 之间有何关联? \\
\hline 络合滴定法 & EDTA滴定中的酸度控制 & $\begin{array}{l}\text { 为什么在络合滴定中一定要控制酸度? 不控制会出现怎样的结果? 对缓冲溶液的要求 } \\
\text { 有哪些? }\end{array}$ \\
\hline $\begin{array}{l}\text { 氧化还原滴 } \\
\text { 定法 }\end{array}$ & 条件电极电位 & $\begin{array}{l}\text { 多数高锰酸钾溶液参与的反应都是生成 } \mathrm{Mn}^{2+} \text {, 但是高锰酸钾溶液放置久了会有沉淀, } \\
\text { 为什么? 滴定终点通常是溶液呈现粉红色, 放置一会儿颜色会消失, 为什么 } ?\end{array}$ \\
\hline 沉淀滴定法 & 沉淀滴定法应用 & $\begin{array}{l}\text { 化学反应中沉淀反应很多, 是不是都可以用来进行定量滴定分析? 钻尔奇钻片的主要 } \\
\text { 成分是碳酸钙, 摄入后为什么能被身体吸收呢? }\end{array}$ \\
\hline 分光光度法 & 物质的紫外-可见光吸收 & $\begin{array}{l}\text { 紫外-可见光谱是鉴别珠宝的一种方法, 这利用了什么原理? 饮用水的 } \mathrm{Fe} \text { 离子含量和空 } \\
\text { 气中的甲醛含量都可以利用分光光度法来测量, 对于颜色不明显的物质的定量检测是 } \\
\text { 如何实现的? }\end{array}$ \\
\hline
\end{tabular}

除了围绕理论教学内容设置的 “问题” 和案例分析外, 我们还设置了一些需要综合运用分析化 学理论和先修课程知识的分析思考题作为综合作业供学生分组完成, 来培养学生提炼科学问题和进 行初步科学研究的能力。比如, 在学习完四大滴定内容后, 鼓励学生从生活中选择合适的可以采用 滴定分析方法的综合性问题, 例如鸡蛋壳中碳酸钙的含量、西红柿中 $\mathrm{Vc}$ 的含量、柠檬中柠檬酸的含 量分析等, 进行文献调研、方案设计、可行性分析以及实验设计。这些问题是开放的, 仅要求学生 采用四大滴定的理论方法进行实验设计。学生每 4 人一组, 通过分工协作、小组讨论, 完成相关的设 计后, 在课上选派代表进行答辩研讨。经过几年的实践, 学生反映这样的综合作业做起来很有意思, 做作业过程中不仅梳理了相关的概念和知识点, 分析和解决问题的能力也得到了提高。从学生课堂 讨论中, 我们也发现他们不仅思考如何利用所学理论设计实验, 也开始区分不同的实验设计之间的 差别和特色。

\section{2 丰富教学形式, 调动学生主动参与}

强化基础是教学的根本要求, 激发兴趣是提升教学效果的手段。在此基础上引导学生主动学习 和探索未知才能实现拔尖创新人才培养的目标。为了实现人才培养目标和 “在用中学、在学中用” 的教学目的, 我们也在组织教学过程中将多种教学形式结合起来, 采用启发、讨论、参与等形式, 激发学生的学习兴趣, 从而更有效地引领学生主动思考, 调动学生的学习主动性。核心内容的教学 我们主要采用 “问题” 导向的教学模式, 包括课堂讨论、问答等形式。在教学设计上, 对于与先修 
课程关系比较密切的教学内容, 则考虑让学生独立去回顾先修内容, 通过问答来检验效果, 而在课 堂上则重点组织学生去讨论其中的分析化学内涵和处理思路, 更多地去引导学生思考 “为什么要这 么做, 这么做会有什么好处, 其他方法会有什么问题”。例如: 当讲述化学分析中 $60 \%-70 \%$ 的误差 来源于采样和预处理的过程后, 让学生讨论分析化学实验中的每个环节对于获取正确结果的必要性 和重要性, 实现 “在用中学”。这样以课堂教学内容为点, “以点及面”, 不仅帮助学生梳理知识 结构, 也在比较和分析中引导他们去主动地思考和理解教学内容中包含的知识点。对于相对独立的 分析化学理论内容, 详细讲解其中的来龙去脉, 通过 “问题” 引导学生的思维与教学内容产生共鸣, 确保教学效果。针对重点教学内容, 我们也会收集整理发表在Science、Nature等期刊上应用到化学 分析理论和方法的文献作为 “问题” 进行教师讲读和 “翻转课堂”, 让学生了解化学学科和化学分 析的博大精深和旺盛的生命力。对于综合作业和实验设计, 则要求学生针对具体问题去搜集相关资 料, 进行阅读、分析、讨论和归纳、撰写报告和答辩, 通过这些过程, 在加深学生对教学内容理解 的同时, 培养他们严谨的科学态度和团队精神。

\section{3 渗入思政元素, 增强学生责任意识}

分析化学作为应用化学专业的一门核心课程, 也是一门实践科学, 与人们衣食住行紧密相关。 如果能够在教学中适时恰切地渗入思政元素, 则可以形成协同育人效应, 让学生在认真听课的过程 中能够深刻地理解相关知识点, 更能润物无痕地培养他们的责任意识和批判性思维能力。在教学过 程中, 我们一方面加入科学家的传记, 利用科学家真实经历去引导学生树立正确的科学观、世界观、 道德观和人生观。比如, 在误差及分析数据的统计处理部分, “有效数字” 是重点教学内容, 而且 “树立量的概念” 是分析化学学科的重要要求。我们在讲述过程中会介绍 “瑞利的故事一一树立量的 概念的重要性” “卢嘉锡与小数点的故事” 等, 结合这些名人轶事阐明 “量” 的概念和重要性, 同 时也培养学生实事求是、认真严谨的科学态度和思维方法 ${ }^{[16,17]}$ 。另一方面, 我们也在课堂中引入与 教学内容相关的重要时政事件。例如, 在介绍酸碱滴定的应用一一化合物中氮含量的测定(凯氏定氮 法)时, 会提及2008年席卷全国的三聚氰胺 “毒奶粉事件” , 让学生更深刻地认识 “蛋白质定量分析 方法” 本身的缺陷, 以及熟悉该方法的相关人员职业道德缺失给社会带来的严重后果。在讲授氧化 还原滴定的应用一一化学需氧量(COD)的高锰酸钾测定法时, 会与学生讨论我国的环境现状, 使学生 认识到环境污染问题既需要有关部门利用分析化学的手段加强环境监测、建立完善的法律法规强化 监管, 也需要提高群众的社会责任意识与家国情怀, 牢记 “绿水青山才是金山银山”。这样的教学, 不仅强化了学生对理论知识的理解, 也培养了他们的道德意识和家国情怀。

\section{4 完善考评方式, 提高学生综合素质}

为了体现学生在整个教学过程中的参与情况和对教学内容的理解掌握程度, 我们对课程的考核 方式也进行了改进。我校应用化学理科专业的分析化学是必修课, 期末考试是闭卷考试并占总评成 绩的 $70 \%$ 。为充分体现学生在教学过程中的参与度和成果, 平时成绩由课内讨论互动 $(5 \%)$ 、随堂测 试 $(5 \%)$ 、作业 $(10 \%)$ 、综合作业与实验设计 $(10 \%)$ 等几部分组成。对于课内讨论互动, 助教会在每次 课上记录学生提问和参与讨论的次数和情况, 量化后作为期末总评时统计分档的依据。综合作业和 实验设计我们实行 “三选一”, 即学生根据兴趣从三道题选作一道题。因为学生综合作业和实验设 计选题难度不完全相同, 目前主要由教师根据作业撰写和答辩情况 $(2 \%)$ 、理论运用的合理性 (4\%)、 方案可行性(2\%)和完整性(2\%)进行分档打分。考试结束后, 我们也会邀请班干部和部分同学进行座 谈, 了解他们对课程的真实感受, 倾听他们的心声, 从而进一步改善课程教学。从近几年的教学和 学生反馈情况来看, 现行考评方式可以较为客观地反映学生的学习效果。我们也正在建设与现行教 学内容配套的新媒体平台和量化指标体系, 以实现对学生学习过程的动态监测。 


\section{5 结语}

这几年里, 针对拔尖创新人才培养的要求, 我们在分析化学教学中通过优化教学内容, 实施 “以 学生为中心” “问题为导向” 的教学设计, 让学生体验 “在学中用” “在用中学”, 取得了师生满 意的教学效果。但是, 在实施过程中依然面临着诸多挑战。一方面, 对教师有了更高的要求, 因为 这种授课模式需要教师投入较多的时间和精力, 而且要不断更新、完善教学内容和自己的知识体系。 对学生来说, 媒体技术的快速发展也带来了学习方法和知识获取途径的变革。尽管主动学习过程充 满挑战和趣味性, 但也需要较好的自学能力和一定的精力投入, 这对青年学生来说也是一个挑战, 需 要教师的督促和激励。今后工作中, 我们将进一步优化和改进教学内容, 紧跟时代步伐, 针对前沿 发展和学科领域特点及时更新教学内容, 进一步提高分析化学课程的教学效果和水平。

\section{参 考 文 献}

[1] 蔡苹, 胡锴, 罗威, 邓鹤翔, 程功録. 大学化学, 2019, 34 (10), 41.

[2] 徐孝菲, 沈宏, 王敏, 胡吉明. 大学化学, 2019, 34 (10), 45.

[3] 马晓爽, 苏燕, 姜林, 张放, 钟欣芮, 余孝其, 李梦龙, 郑成斌. 大学化学, 2019, 34 (10), 74.

[4] 李维红, 张奇涵, 李娜, 王颖霞, 裴坚. 大学化学, 2019, 34 (10), 1.

[5] 王佰全. 大学化学, 2019, 34 (10), 18.

[6] 魏䍭, 李运超, 范楼珍. 大学化学, 2019, 34 (10), 71 .

[7] 黎朝, 温庭斌, 吴伟泰, 张延东, 曹晓宇, 朱亚先, 吕釒. 大学化学, 2021, 36 (5), 2010002.

[8] 叶芳, 王佰全, 李一峻, 郭东升. 大学化学, 2021, 36 (5), 2008058.

[9] 李永军, 王玉枝, 张晓兵, 范媛吉, 蔡炽, 陈淑云, 吴英鹏. 大学化学, 2021, 36 (5), 2008022.

[10] 韦骅峰, 季斑希. 中国高教研究, 2021, No. 6, 30.

[11] 徐首红, 张文清, 徐志珍, 罗千福, 刘洪来. 大学化学, 2019, 34 (9), 21.

[12] 纪敏, 孟长功, 姜文风, 吴硕, 赵艳秋. 大学化学, 2021, 36 (5), 2003036.

[13] 张树永, 朱亚先. 大学化学, 2017, 32 (1), 9 .

[14] 朱亚先, 张树永. 中国大学教学, 2015, No. 2, 31 .

[15] 王玉枝, 李颖, 杨屹, 宦双燕, 吴硕, 文志宁, 苏星光, 张文清. 大学化学, 2019, 34 (4), 39

[16] 王焕锋, 李玉玲, 王利平, 李晓静, 李靖靖, 王岗. 大学化学, 2021, 36 (9), 2102038.

[17] 盛寒䒯, 张波, 张文清, 田佳, 赵怡, 夏玮, 刘海燕, 钱俊红, 王氢, 章弘扬, 等. 大学化学, 2021, 36 (9), 2102019. 